\title{
toponymie et géotechnique
}

\author{
par \\ P. Habib \\ Président du Comité Français de Mécanique des Sols
}

\begin{abstract}
L'origine des noms des lieux permet de découvrir des traces d'un passé venu jusqu'à nos jours. Ces noms peuvent provenir d'une multitude de choses: village, habitat isolé, nom de personne, topographie, végétation, etc. Les activités humaines, la propriété, le culte et l'observation de la nature y sont évidemment prépondérants. Peut-on trouver dans ces indications anciennes des informations qui peuvent être utiles aux géotechniciens? Par exemple, Balme, Barme, Baume, Baumette, mots d'origine probablement ligure, indiquent la présence d'une grotte; peut-on en induire qu'on se trouve dans une zone de calcaire karstique? ou plus modestement qu'une Baume dans un calcaire doit faire penser à un karst? Voici un autre exemple; si on examine les lieux-dits sur une carte à petite échelle, on voit apparaître la répétition d'expressions, peut-être avec des variations orthographiques, mais avec la même consonance; peut-on en trouver quelques-unes qui ont rapport avec le sol?
\end{abstract}

A ce titre, la région des Alpes en France est particulièrement intéressante, car elle a toujours été un carrefour d'influences; le relief ouvert de ses vallées en a fait un lieu de passage et d'invasion; des influences celtes, gallo-romaines, mérovingiennes, germaniques et même arabes peuvent y être retrouvées, même si les déformations sémantiques ont rendu la plupart du temps les mots méconnaissables. En effet, la dérive des parlers locaux, maigré la stabilité des populations de montagnes, plus accrochées que d'autres à leur relief et en général épargnées des grandes mouvances militaires, s'est chargée de modifier peu à peu les vocables suivant des règles malgré tout compréhensibles et assez fidèles, mais difficiles à interpréter ou à imaginer.

Ces influences nombreuses entraînent dans les Alpes la cohabitation de séries différentes de mots et ainsi pour la rivière, le Doron ligure ne peut pas être confondu avec la rivière celtique: Carus, Cher, Fier, Chier, Guiers, Sierroz, ni avec le torrent germain : Bac, Bach, Bec, Bay, Baye, ni avec l'eau latine: Aix, Eve,
Evette, Aigues, Aiguier, Agal, Arve ou la rivière latine : Rivus, Rieu, Ru. La toponymie alpine est donc particulièrement riche, mais ses enseignements s'étendent évidemment à la France entière.

Bien entendu, l'étymologie ne s'improvise pas et il faut se garder des ressemblances et des à peu près : Le plan ou La plane, c'est la plaine, mais Le plane ou le Le planey est le lieu où poussent... les platanes; Les avanchers est un lieu où pousse l'osier (donc un lieu humide), mais Le lavancher est l'endroit où passe l'avalanche... L'étymologie naïve a entraîné les plus grands, dont Littré, dans des voies sans issues. Actuellement, l'étymologie est une science bien aquise et la toponymie donne une base solide à de telles recherches et comparaisons, par l'étude des documents les plus anciens possibles pour remonter au plus loin et utiliser ensuite l'analogie et les modes classiques de dérivation. Les dictionnaires toponymiques ainsi établis (Dauzat et Al., 1978 - Gros, 1973) sont alors des outils de travail efficaces, et il n'est pas trop aventuré d'explorer les noms de lieux pour tirer des renseignements sur la nature des accidents de terrain et à ce titre pour apporter des informations utiles à la géotechnique, ou tout au moins des éléments permettant d'orienter une première reconnaissance.

Une des origines les plus fréquentes de noms de lieux - qui ne nous intéresse guère ici, sauf par les erreurs qu'elle peut engendrer - est les noms sacrés ou les noms de personnes, d'ailleurs en interaction avec les noms de lieux. Ainsi, un fermier ayant construit un four à pain, sa maison devient la maison du four et le fermier Monsieur Dufour. Lequel Dufour déménage, lui ou son fils, pour s'installer au plein milieu des bois, où son habitation devient à nouveau la maison Dufour. Mais alors, allez donc chercher le four! Les origines qui peuvent nous intéresser sont géologiques, topographiques, géographiques et aussi un peu botaniques. Malheureusement, Porigine des noms est le plus souvent botanique : Chenay, Charnay, Cernay, Chane (chêne), Coudray, Caroley (coudrier), Pece, Pesay, 
Pesey, Pinet, Pignes (pin), Sapey, Sapine, Savine (sapin), Saulce, Sauge, Saussier, Soucy, Sochaux (saule), Fau, Feu, Fou, Fol, Fay, Foi, Fayet, Faou (hêtre), Arbelet, Arblet, Herblay (érable), Boisse, Boissière, Buis, Buisson, Breuil, Brel, Bosquet, Bouchet (bois), etc., ce qui est assez peu enrichissant pour le géotechnicien.

L'origine des noms est parfois topographique ou géographique, ce qui est déjà mieux, mais pas fondamental parce que normalement visible : Endroit, Adroit, Adret (la partie au soleil), Aiguille, Uille, Ulie, Ouille, Huile (la pointe), Entre-deux-giers, Entre-deuxNants (entre deux rivières), Saix, Saxe, Sache, Sachère, Sassière, Sasso (le rocher), Combe, Combel, Combalout (combe à loups), Combefol, Combefort (combe à hêtres), Cormet, Colmet, Quern, Cairn (point culminant; d'où la notion de col). Plagne, Replat, Replaton, Plan, Plat, Table, Tavole, etc.

L'origine des noms de lieux est parfois minéralogique ou signalant l'existence de certains produits, ce qui peut être une information utile, et il faut citer:

Arènes, Arnier, Arenier, Araignée (latin);

Sables, Sablière, Sablon (du latin sabulum, sabulare : lester);

Grave, Gravière, Grenant, Gravelotte (celte);

Glaire, Glière, Glairon (terrains sablonneux);

La Thuile, Les Thuilles, Tuillerie, Tuilière, Terrallière, La Poterie, Félines, Flins (présence de terre à brique);

Ardillat, Ardillières, Argilliers, Argelès, Glaizie, Glaizé (Argile);

La Casse, Clapet, Clapier, Clapière (amas rocheux, terrain rempli de pierres);

Pierre, Petrelle, Perron, Perrière, La Pérouse, Petret, Perret, Calce, Caux (pierres et roches en abondance);

Losa, Lose, Lause, Louse, Lozière (schistes ardoisiers);

Tuf, Touz, Tovet, Tovière (carrière de Tuf), toutes informations qui sont pour la géotechnique plus importantes que les Argentière, Plombière, Ferrière et autres Salins, ou Four, Fourneaux, Fornet (en général d'ailleurs four à chaux, mais parfois d'autres choses).

Finalement, les mots véritablement intéressants pour la Mécanique des Sols ou des Roches, sont très peu nombreux :

- Les sources :

Fons, Font, Fond, Fontaine, Fontanette, Fontenay, Fredière, Evette, Aiguier et Chaudanne, Chaudagne, Choudan, Chaudeau (quand elles sont thermales);

- les mauvais lieux marécageux plats et fangeux, avec de nombreuses séries de vocables généralement bien compréhensibles:

Mouille, Moille, Molie, Mollière, Mollasses (lat. mollia : lieu humide);

Étain, Étang, Estaing (stagnant);

Flachet, Fléchère (germ. flach : plat);

Pallud, Palluet, Épallus, Palluel (lat. paludis : marais);

Sagne, Seigne, Saigne, Sanière (végétation de basfonds);

Roset, Rosey, Roseau, Rosière (roseaux).

- Les lieux escarpés où l'on accède par des chemins taillés dans la pierre: Échelles, Scolette, Échaillet, Échaillon.
- Les roches de mauvaises qualités: Rochemolle, Roche pourrie.

- La montagne qui s'éboule: Ruine, Reine, Ronna, Runa, Ronne.

- Les terrains en pente soutenus par des murs de soutènement (échafaudages): Chaffat, Chaffar, Échaffa, Jaffa, Échapau.

- Les terrains qui glissent (qui coulent): Culée, Culatte, Culaz, Colle, Colette.

- Les terrains qui se rompent ou se ravinent : Rupes, Érupeaux, Érupe, Rupta, Rotte, Route, Roux, Droux; Praz Rot (le pré rompu), Cote Route (la cote rompue).

Tout cela n'est pas grand chose, surtout si l'on constate qu'en plaçant toutes les Baume et autres Balme sur la carte géologique, on voit que des grottes peuvent se produire dans de nombreuses formations et pas uniquement dans les calcaires...

Dernier espoir : on sait qu'il existe des liens entre la végétation et la qualité des terres (Bournieras, 1974). Peut-on en tirer des informations à partir de la toponymie?

C'est bien difficile à dire en zone urbanisée : il y a à peu près aussi longtemps que tous les arbres de ce nom ont disparu d'Aulnay-sous-Bois, que de celui où le sable de la place de Grève a été caché par des pavés. En zone rurale, les travaux de l'agriculture ont complètement modifié les paysages. Par contre, en montagne on peut espérer que la nature n'ait pas été trop perturbée par I'homme. Hélas! La colline du Biollet (bouleau) est couverte d'aulnes, les Bruyères et les Brevières (terrains vagues laissés en friches) sont remplis de sapins, il n'y a plus que des alpages aux Avellane et Olagnier (noisettiers) et il y a des bouleaux au lieu de frênes au Frenay; quant aux charmes, comme il y a eu une grande épidémie l'an dernier en France, il est difficile d'en trouver nulle part, ni au Charmette. Bref, les changements climatiques (probablement vers le chaud) ont considérablement modifié la végétation depuis les temps lointains où les noms se sont fixés.

Au terme de cette longue enquête, il reste peu de choses utilisables si l'on tient compte de la rareté des mots qui intéressent les sols et du danger des faux amis. II nous reste peu de certitudes, si ce n'est que les cimes des Alpes sont blanches, comme les falaises de craie d'Albion, et que rien ne vaut un bon sondage..

\section{Remerciements :}

En terminant cette note, je dois remercier M. E. RECORDON, ancien Président de la Société Suisse de Mécanique des Sols et Professeur à l'École Polytechnique Fédérale de Lausanne, ainsi que $\mathrm{M}^{\text {lle }} \mathrm{C}$. HABIB, Professeur Agrégé, pour différentes conversations et recherches ou envois de documents bibliographiques. 


\section{Références bibliographiques}

M. BOURNIÉRAS (1974), La végétation, ses liens avec le substrat géologique et le sol », dans : Ch. Pomerol et L. Feugueur (1974), “ Guide géologique du Bassin de Paris, lle de France, Pays de Bray", pp. 54-59 (Masson, Paris).

A. DAUZAT, G. DESLANDES, de ROSTAING (1978), " Dictionnaire étymologique des noms de rivières et de montagnes en France " (Klincksieck, Paris).
A. DAUZAT, et Ch. ROSTAING (1978), «Dictionnaire étymologique des noms de lieux en France" (Guénégaud, Paris).

A. GROS (1973), “Dictionnaire étymologique des noms de lieu de la Savoie” (Imprimerie Réunie de Chambéry).

J.J. HISELY (1851), "Histoire du Comté de Gruyère " (G. Bridel, Lausanne). 